\title{
The CERN n_TOF facility: a unique tool for nuclear data mea- surement
}

F. Mingrone ${ }^{1,2, a}$, O. Aberle ${ }^{2}$, J. Andrzejewski ${ }^{3}$, L. Audouin ${ }^{4}$, V. Bécares ${ }^{5}$, M. Bacak $^{6}$, J. BalibreaCorrea $^{5}$, M. Barbagallo ${ }^{7}$, S. Barros ${ }^{8}$, F. Bečvář ${ }^{9}$, C. Beinrucker ${ }^{10}$, E. Berthoumieux ${ }^{11}$, J. Billowes ${ }^{12}$, D. Bosnar ${ }^{13}$, M. Brugger ${ }^{2}$, M. Caamaño ${ }^{14}$, F. Calviño ${ }^{15}$, M. Calviani ${ }^{2}$, D. Cano$\mathrm{Ott}^{5}$, R. Cardella ${ }^{2}$, A. Casanovas ${ }^{15}$, D. M. Castelluccio ${ }^{16,1}$, F. Cerutti ${ }^{2}$, Y. Chen ${ }^{4}$, E. Chiaveri ${ }^{2}$, N. Colonna ${ }^{7}$, M. A. Cortés-Giraldo ${ }^{17}$, G. Cortés $^{15}$, L. Cosentino ${ }^{18}$, L. Damone ${ }^{7}$, M. Diakaki ${ }^{11}$, C. Domingo-Pardo ${ }^{19}$, R. Dressler ${ }^{20}$, E. Dupont ${ }^{11}$, I. Durán ${ }^{14}$, B. Fernández-Domínguez ${ }^{14}$, A. Ferrari2 ${ }^{2}$, P. Ferreira ${ }^{8}$, P. Finocchiaro ${ }^{18}$, V. Furman ${ }^{21}$, S. Ganesan ${ }^{22}$, A. A. Garcia-Rios ${ }^{5}$, A. Gawlik ${ }^{3}$, I. Gheorghe ${ }^{23}$, T. Glodariu ${ }^{23}$, I. F. Gonçalves ${ }^{8}$, E. González ${ }^{5}$, A. Goverdovski2 ${ }^{24}$, E. Griesmayer ${ }^{6}$, C. Guerrero ${ }^{17}$, F. Gunsing ${ }^{11,2}$, K. Göbel ${ }^{10}$, H. Harada ${ }^{25}$, T. Heftrich ${ }^{10}$, S. Heinitz ${ }^{20}$, J. Heyse ${ }^{26}$, G. Jenkins ${ }^{27}$, E. Jericha ${ }^{6}$, F. Käppeler ${ }^{28}$, Y. Kadi², T. Katabuchi29, P. Kavrigin ${ }^{6}$, V. Ketlerov 24 , V. Khryachkov ${ }^{24}$, A. Kimura ${ }^{25}$, N. Kivel ${ }^{20}$, M. Kokkoris ${ }^{30}$, M. Krtička ${ }^{9}$, E. LealCidoncha $^{14}$, C. Lederer ${ }^{31,10}$, H. Leeb 6 , J. Lerendegui ${ }^{17}$, S. Lo Meo ${ }^{16,1}$, S. Lonsdale ${ }^{31}$, R. Losito ${ }^{2}$, D. Macina ${ }^{2}$, J. Marganiec ${ }^{3}$, T. Martínez ${ }^{5}$, C. Massimi ${ }^{1,32}$, P. Mastinu ${ }^{33}$, M. Mastromarco ${ }^{7}$, F. Matteucci ${ }^{34}$, E. A. Maugeri ${ }^{20}$, E. Mendoza ${ }^{5}$, A. Mengoni ${ }^{16}$, P. M. Milazzo ${ }^{34}$, M. Mirea ${ }^{23}$, S. Montesano ${ }^{2}$, A. Musumarra ${ }^{18}$, R. Nolte ${ }^{35}$, A. Oprea $^{23}$, N. Patronis ${ }^{36}$, A. Pavlik ${ }^{37}$, J. Perkowski ${ }^{3}$, J. Praena ${ }^{17}$, J. M. Quesada ${ }^{17}$, K. Rajeev ${ }^{22}$, T. Rauscher ${ }^{38,39}$, R. Reifarth ${ }^{10}$, A. Riego-Perez ${ }^{15}$, P. Rout $^{22}$, C. Rubbia ${ }^{2}$, J. A. Ryan ${ }^{12}$, M. Sabaté-Gilarte ${ }^{2,17}$, A. Saxena ${ }^{22}$, P. Schillebeeckx ${ }^{26}$, S. Schmidt ${ }^{10}$, D. Schumann ${ }^{20}$, P. Sedyshev ${ }^{21}$, A. G. Smith ${ }^{12}$, A. Stamatopoulos ${ }^{30}$, G. Tagliente ${ }^{7}$, J. L. Tain $^{19}$, A. Tarifeño-Saldivia ${ }^{19}$, L. Tassan-Got ${ }^{4}$, A. Tsinganis ${ }^{30}$, S. Valenta ${ }^{9}$, G. Vannini ${ }^{1,32}$, V. Variale ${ }^{7}$, P. Vaz ${ }^{8}$, A. Ventura ${ }^{1}$, V. Vlachoudis ${ }^{5}$, R. Vlastou ${ }^{30}$, A. Wallner ${ }^{40}$, S. Warren ${ }^{12}$, M. Weigand ${ }^{10}$, C. Weiss ${ }^{2,6}$, C. Wolf ${ }^{10}$, P. J. Woods ${ }^{31}$, T. Wright ${ }^{12}$, and P. Žugec ${ }^{13,2}$

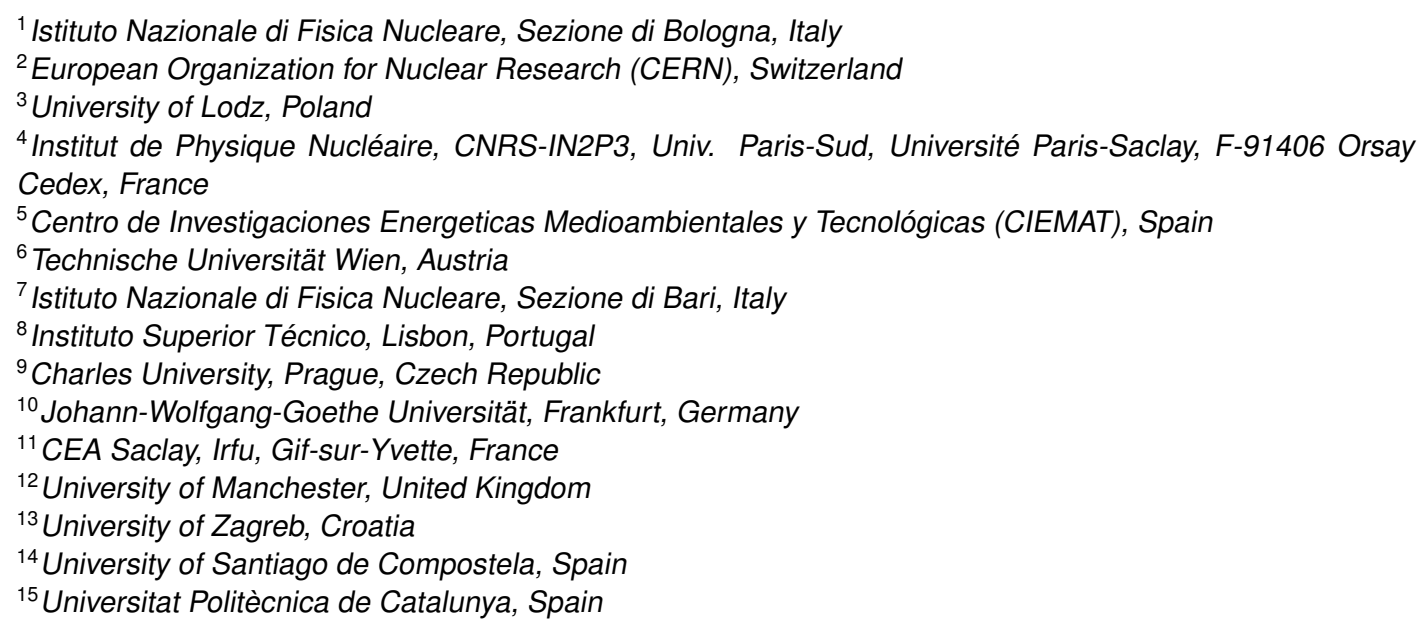

a e-mail: federica.mingrone@cern.ch 


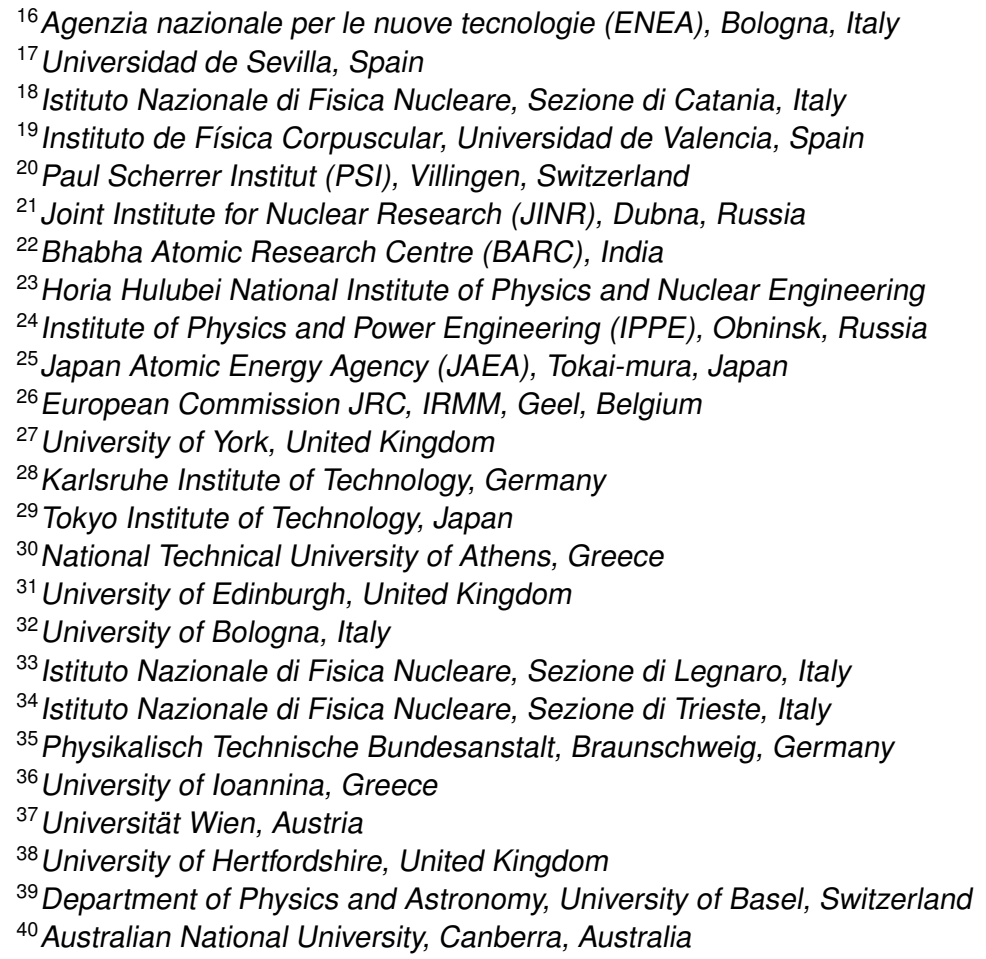

Abstract. The study of the resonant structures in neutron-nucleus cross-sections, and therefore of the compound-nucleus reaction mechanism, requires spectroscopic measurements to determine with high accuracy the energy of the neutron interacting with the material under study.

To this purpose, the neutron time-of-flight facility n_TOF has been operating since 2001 at CERN. Its characteristics, such as the high intensity instantaneous neutron flux, the wide energy range from thermal to few $\mathrm{GeV}$, and the very good energy resolution, are perfectly suited to perform high-quality measurements of neutron-induced reaction cross sections. The precise and accurate knowledge of these cross sections plays a fundamental role in nuclear technologies, nuclear astrophysics and nuclear physics.

Two different measuring stations are available at the n_TOF facility, called EAR1 and EAR2, with different characteristics of intensity of the neutron flux and energy resolution. These experimental areas, combined with advanced detection systems lead to a great flexibility in performing challenging measurement of high precision and accuracy, and allow the investigation isotopes with very low cross sections, or available only in small quantities, or with very high specific activity.

The characteristics and performances of the two experimental areas of the $\mathrm{n}_{-} \mathrm{TOF}$ facility will be presented, together with the most important measurements performed to date and their physics case. In addition, the significant upcoming measurements will be introduced. 


\section{Introduction}

The time-of-flight facility of CERN, called n_TOF, became operative in 2001 based on an idea by Rubbia et al. [1], and since then it occupies a major role in the field of neutron cross-section measurements. In particular, thanks to the broad energy spectrum covered and the possibility to use the time-of-flight (TOF) technique to precisely select the incident neutron energy, high-resolution neutron spectroscopic measurements are possible. The pulsed neutron beam at n_TOF is produced by spallation of $20 \mathrm{GeV} / \mathrm{c}$ protons from the CERN Proton Synchrotron accelerator on a water-cooled $\mathrm{Pb}$ target [2]. The pulsed neutron source is used together with a moderation system, so that the n_TOF neutron beam covers about eleven orders of magnitude in energy from thermal to GeV. A scheme of the facility is given in Fig. 1. The spallation mechanism is a remarkably powerful source of neutrons, and at the proton energy of $20 \mathrm{GeV}$ about 300 neutrons per proton are produced in the $\mathrm{n}$-TOF target. The instantaneous intensity of the n_TOF neutron output is therefore one of the highest among the TOF facilities.

After a phase of design and feasibility studies in the late 90 s, the commissioning of the facility started in 2000 and the first phase of measurements ran from 2001 to 2004. In this period of time, called n_TOF Phase-1, $25(\mathrm{n}, \gamma)$ and $12(\mathrm{n}, f)$ measurements have been successfully performed. In 2004 a technical problem related with the spallation target interrupted the data-taking for four years, during which the target was re-designed and replaced, and improvements such as the upgrade of EAR1 to a Class-A laboratory were implemented. From the end of 2008 till the end of 2012 the facility resumed operation in its Phase-2, during which $14(\mathrm{n}, \gamma)$ and $3(\mathrm{n}, f)$ measurements have been performed, together with the first two (n,cp) measurements. During the Long Shutdown 1 (LS1) of CERN [3] a second short flight-path [4], complementing the existing $185 \mathrm{~m}$ one, has been constructed and completed on the 25 th of July 2014 when the n_TOF Phase-3 started. Since then, in this new experimental area (EAR2) one (n, $\gamma)$, two $(n, f)$ and two (n,cp) measurements have been successfully
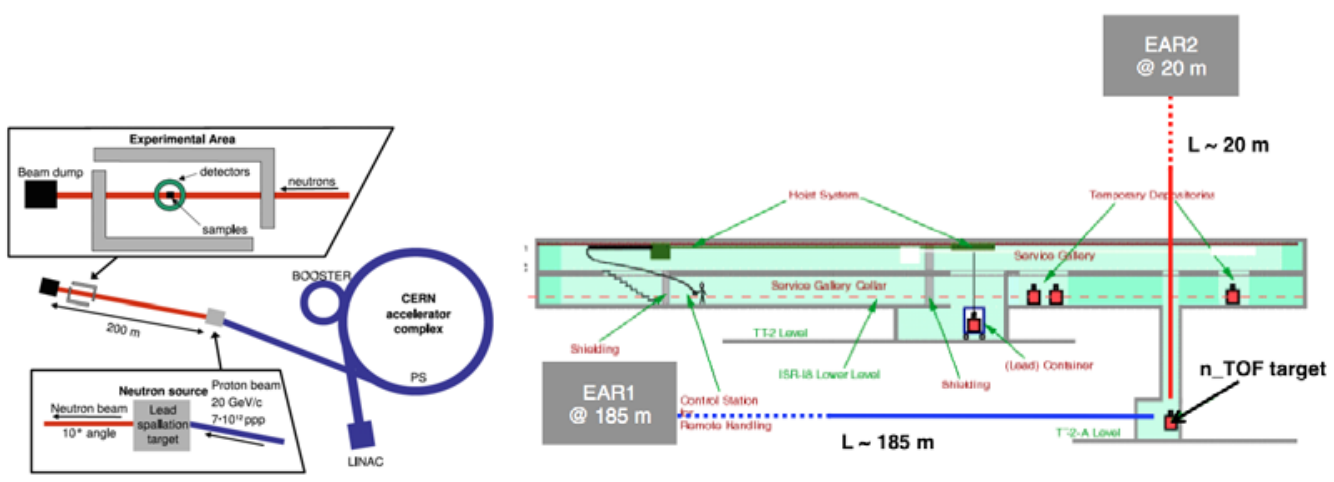

Figure 1. Left: Layout of the $n \_$TOF facility within the CERN accelerator complex [5]. The LINAC feeds the PS-Booster, which provides the PS with protons of $1.4 \mathrm{GeV} / \mathrm{c}$ for acceleration up to $20 \mathrm{GeV} / \mathrm{c}$. This beam is extracted and sent to the $\mathrm{n} \_$TOF lead spallation target in bunches of $7 \times 10^{12}$ protons. The experimental hall is located near the end of the $200 \mathrm{~m}$ long neutron beam line. Right: Schematic of the two different beam lines of the $\mathrm{n}_{-}$TOF facility. The first experimental area (EAR1) is located horizontally at a distance of $185 \mathrm{~m}$ from the neutron source, while the new experimental area (EAR2) is located at $90^{\circ}$ with respect to the proton beam direction with a flight-path of about $20 \mathrm{~m}$. 
completed, together with the seven $(\mathrm{n}, \gamma)$ and one $(\mathrm{n}, f)$ measurements performed in parallel at the first measuring station (EAR1).

\section{The $n \_$TOF neutron beam}

Two different spallation targets have been used at n_TOF as neutron-producing targets. The first one, composed of $\mathrm{Pb}$ blocks rectangular in shape and cooled by a $5 \mathrm{~cm}$ water layer acting also as the moderator of the neutron spectrum, had to be replaced after four years of operation due to damages caused in some spots by inefficient cooling. The second target, installed in 2008, was equipped with a more efficient cooling system based on recirculating water, and with a separate moderator circuit to permit the use of different moderating materials. This target is made of a monolithic cylindrical block of lead, $40 \mathrm{~cm}$ in length and $60 \mathrm{~cm}$ in diameter. On the neutron exit face of the target opposite to the proton entrance, cooling is ensured by a water layer of $1 \mathrm{~cm}$ thickness, while moderation of the neutron spectrum is performed with a $4 \mathrm{~cm}$ thick layer of either normal, heavy or borated water. Borated water is now mostly being used to minimize the production of $2.2 \mathrm{MeV}$ in-beam $\gamma$ rays from $n+\mathrm{H} \rightarrow{ }^{2} \mathrm{H}+\gamma$ reactions, that constitutes the main source of background in measurements of capture cross-sections in the $\mathrm{keV}$ neutron-energy region.

The innovative features of the n_TOF neutron beam derive from the characteristics of the pulsed proton-beam from the CERN Proton-Synchrotron (PS), consisting of bunches with a high momentum of $20 \mathrm{GeV} / \mathrm{c}$, a high peak current of $7 \times 10^{12}$ protons per bunch and a low duty cycle of 0.5 $\mathrm{Hz}$, thus avoiding pile-up of events coming from consecutive bunches and resulting in the very high intensity of the n_TOF neutron source, of the order of $10^{4}$ neutrons $/ \mathrm{cm}^{2} /$ pulse in EAR1 and $10^{6}$ neutrons $/ \mathrm{cm}^{2} /$ pulse in EAR2.

Two different beam lines originate from the spallation target: one horizontal, 200-m long, which leads to the first experimental area, and one vertical, 20-m long, which ends in the second experimental area. Along both the beam lines a sweeping magnet is placed to deflect the remaining charged particles in the beam, and the tube has to cross various shielding elements that stop particles travelling around the beam. Two collimators are used to shape the neutron beam, and a filter station allows to insert black-resonance materials in the beam to accurately evaluate the background in the experimental areas. The complete descriptions of the two beam lines can be found in Ref. [6, 7].

At the beginning of the second experimental campaign (n_TOF Phase-2), the first experimental area was upgraded to a Work Sector Type A, with a series of safety and monitoring systems, in order to allow measurements of high-activity samples without certified sealing. Moreover, the second experimental area has been built as a Class-A laboratory. This key modification was essential to exploit the full potential of the facility.

A fundamental piece of the puzzle of high precision measurements of reaction cross-sections is the point-wise knowledge of the incident neutron flux as a function of energy. The knowledge of its energy dependence is as much important as knowing its absolute value, considering that often cross sections are measured relative to some well known quantity in a particular energy range, as for example a standard cross section, the cross section value at thermal energies or the capture yield at the top of a well isolated saturated resonance.

At $n \_$TOF the neutron flux was experimentally determined using several detection systems based on different principles and reactions. A large effort was devoted to minimize all possible uncertainties, both systematic and statistical, in particular on the energy dependence of the neutron flux. All details can be found in Ref. [8].

Starting from 2010, the neutron flux in EAR1 has been moderated by borated water, while for the previous years normal water has been used. Since the separated moderator circuit is present only on 


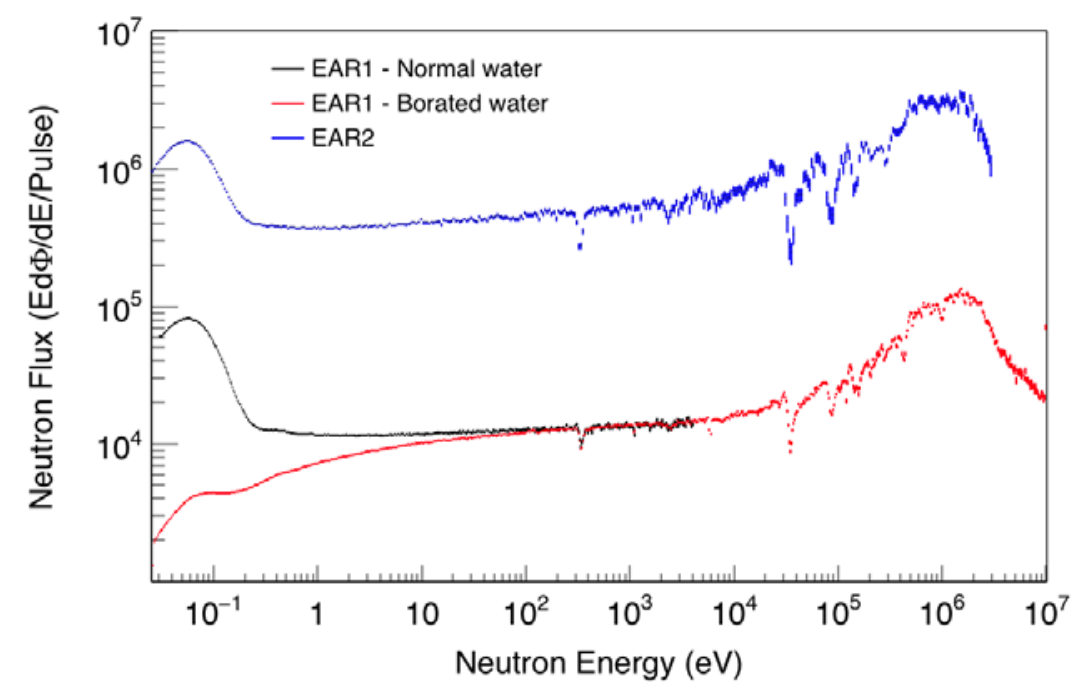

Figure 2. n_TOF neutron flux at EAR1 with normal (black) and borated (red) water as moderator compared with the neutron flux at EAR2 (blue).

the neutron exit face of the target opposite to the proton entrance, the neutron beam arriving in EAR2 can be moderated only by normal water. The effect of the ${ }^{10} \mathrm{~B}$-loaded moderator is a strong reduction of the thermal peak, which changes also with time depending on the ${ }^{10} \mathrm{~B}$ concentration. Above a few hundred $\mathrm{eV}$, the flux does not depend on the moderator anymore and, as expected, is found to be constant over time. In Fig. 2 the flux in EAR1 with different moderators is compared with the flux in EAR2. The intensity of the neutron flux in EAR2 is about 40 times higher than in EAR1 for neutron energies above $10 \mathrm{eV}$, while for lower energies, where the borated water is strongly reducing the flux in EAR1, it is about 500 times higher. On the other hand, it is important to point out that, because of the kinematics within the spallation target, a notable amount of neutrons arrive in EAR2 only for energies below about $300 \mathrm{MeV}$, while in EAR1 (the beam line in the direction of the incoming proton beam) neutron energies up to $1 \mathrm{GeV}$ can be reached.

Another cardinal characteristic of the $n \_$TOF facility is its energy resolution. Being a time-offlight facility, the neutron kinetic energy is calculated from the time taken by the neutrons to travel the distance between the spallation target and the sample under analysis. Because of the neutron transport in the spallation target and in the moderator circuit, and the pulse width of the primary proton beam, the relation between TOF and $E_{n}$ is represented by distributions of a particular shape that depends on the neutron energy. These distributions form the resolution function, i.e. the probability that a neutron with energy $E_{n}$ is detected with a corresponding time of flight $t$, of the n_TOF spectrometer.

The resolution function changes significantly from EAR1 and EAR2, because of its strong dependance on the neutron transport within the target and on the neutron propagation to the experimental areas. Since these effects cannot be experimentally measured, the two resolution functions for the spallation target-moderator assembly have been simulated with FLUKA [9] and MCNP [10] Monte Carlo codes for both the configuration with normal and borated water as moderator material, and neutrons have been propagated through the two different beam lines. In Table 1 the comparison between the resolution broadening as function of neutron energy in EAR1 and EAR2 shows that the energy resolution in EAR2 is limited in resolving individual resonances at high energies compared to EAR1. 
Table 1. The energy resolution as function of neutron energy for EAR1 with borated water as moderator [6] and EAR2 [11].

\begin{tabular}{lll}
\hline$E_{n}$ & \multicolumn{2}{c}{$\Delta E / E$} \\
& EAR1 & EAR2 \\
\hline $1 \mathrm{eV}$ & $3.2 \times 10^{-4}$ & $4.8 \times 10^{-3}$ \\
$10 \mathrm{eV}$ & $3.2 \times 10^{-4}$ & $5.7 \times 10^{-3}$ \\
$100 \mathrm{eV}$ & $4.3 \times 10^{-4}$ & $8.1 \times 10^{-2}$ \\
$1 \mathrm{keV}$ & $5.4 \times 10^{-4}$ & $1.4 \times 10^{-2}$ \\
$10 \mathrm{keV}$ & $1.1 \times 10^{-3}$ & $2.3 \times 10^{-2}$ \\
$100 \mathrm{keV}$ & $2.9 \times 10^{-3}$ & $4.6 \times 10^{-2}$ \\
$1 \mathrm{MeV}$ & $5.3 \times 10^{-3}$ & $5.6 \times 10^{-2}$ \\
\hline
\end{tabular}

With the characteristics listed above n_TOF represents a unique facility for measurements of radioactive isotopes and very low cross-sections, as for example capture cross section of s-process branching-point isotopes, or capture and fission cross-sections of actinides. Depending on the requirements of the reaction under study (e.g. the need of high energy resolution or rather of high neutron flux), the two experimental areas of the n_TOF facility provide a remarkable flexibility to select the best configuration for successful measurement. To fully exploit this potential, different detection systems are available and will be described in the next Section.

\section{Detection Systems}

Several detection systems can be used for neutron induced reaction measurements at the n_TOF facility. Among them, the most significant ones for neutron induced capture, fission and (n,cp) reaction measurements will be here presented.

For capture measurements, two different detection systems have been set up: an array of deuterated liquid scintillator detectors $\left(\mathrm{C}_{6} \mathrm{D}_{6}\right)$ and a $4 \pi \mathrm{BaF}_{2}$ Total Absorption Calorimeter (TAC), both shown in Fig. 3. The first apparatus is characterized by a low sensitivity to background signals induced by scattered neutrons, and it is particularly suitable for measurements of light isotopes for which the elastic scattering cross-section could be several orders of magnitude higher than for capture. An array of four custom made $\mathrm{C}_{6} \mathrm{D}_{6}$ scintillators at the cutting edge of present technology [12] are used at $\mathrm{n}_{-}$TOF in both experimental areas. For highly radioactive and fissile isotopes, in particular for minor actinides, capture measurements are performed in EAR1 with the TAC, which permits the identification of capture events from competing reactions by reconstructing the total energy of the $\gamma$-ray cascade. The relatively large neutron sensitivity of the apparatus is reduced by hardware expedients, as described in Ref. [13]. Recently, the simultaneous measurement of neutron-induced capture and fission reactions have been tested using a MicroMegas [14] detector in combination with the TAC, in order to accurately disentangle the two types of reactions [15]. This method, which has been tested with a measurement of ${ }^{235} \mathrm{U}$ in 2012 , allows accurate capture cross-section measurements of fissile isotopes.

Different detection systems are exploited also for fission cross-section measurements. A multistack Fission Ionization Chamber (FIC), used in the first campaign, has been replaced in Phase-2 by a high-performance MicroMegas detector $[14,16]$ characterized by a better signal-to-noise ratio and has been successfully used in both experimental areas. A second method used at $n \_$TOF relies on the detection of both fission fragments in coincidence. To this purpose, a stack of position-sensitive Parallel Plate Avalanche Counters (PPACs) is used, which also determines the angular distribution 

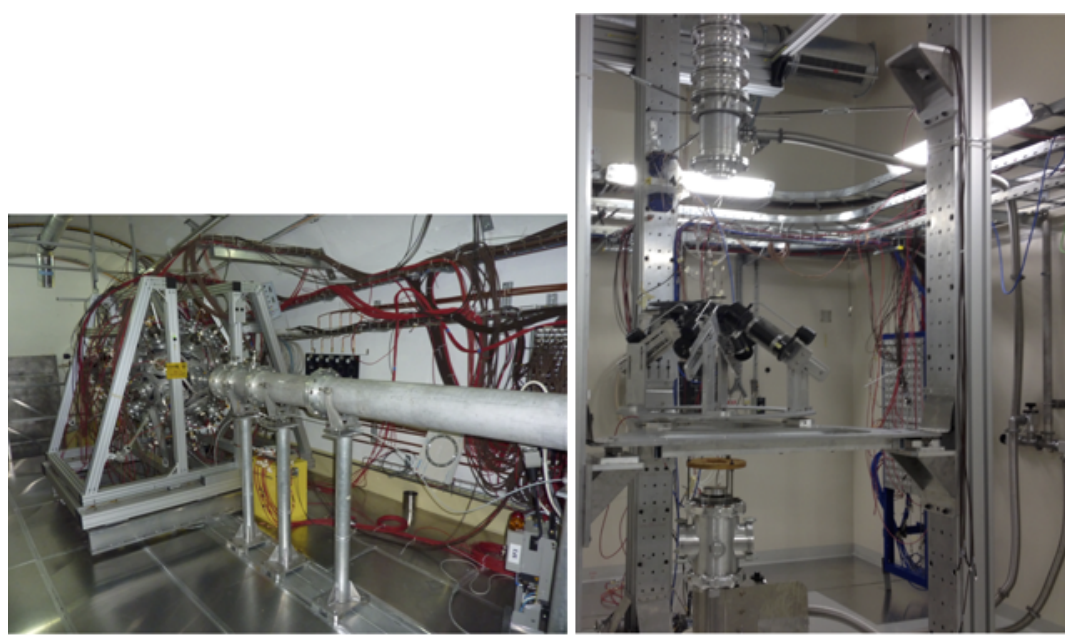

Figure 3. Total absorption calorimeter (TAC) in the $\mathrm{n}_{-}$TOF-EAR1 (left panel) and $\mathrm{C}_{6} \mathrm{D}_{6}$ scintillators set-up in $\mathrm{n} \_$TOF-EAR2 (right panel). The horizontal and vertical beam lines are visible as well.

of the fission fragments $[17,18]$. In all fission measurements, the ratio method is applied, where the cross section of a given isotope is determined relative to the established standard ${ }^{235} \mathrm{U}$. To this purpose, reference samples of ${ }^{235} \mathrm{U}$ are always mounted in the detector in use and measured simultaneously.

Another important set of measurements deals with the (n,cp) reactions, which has become particularly notable thanks to the characteristics of EAR2 that allows one to measure very low cross sections. These measurements can be performed with MicroMegas or Silicon-based detectors. Recently, a new concept of Si-based detector has been developed with the sample sandwiched between two silicon detectors that exploits the emission of back-to-back reaction products together with the coincidence technique to reject the background [19].

\section{The $n \_$TOF facility for science and technology}

As previously pointed out, nuclear reactions induced by neutrons play a fundamental role in nuclear technology, being at the basis of reactor physics, and are of great importance in other fields such as Nuclear Astrophysics and fundamental Nuclear Physics. Many of the existing experimental data have been evaluated and made available through nuclear data libraries and databases. Nevertheless, new developments in emerging nuclear technologies, as well as the newest astrophysical and nuclear models, require more precise and accurate data together with new measurements for a large number of isotopes.

For nuclear technologies, the prediction of the behaviour of reactor cores depends strongly on capture and fission cross-section data. In particular, the R\&D of new reactor concepts, as GenerationIV [20] and Accelerator Driven System (ADS) [21], require precise and accurate measurements of neutron capture and fission cross sections of actinides in the thermal $(\mathrm{meV})$, epithermal $(\mathrm{eV}-\mathrm{keV})$ and fast $(\mathrm{MeV})$ energy regions. At $\mathrm{n}_{-} \mathrm{TOF}$ different measurements of reaction cross-sections addressed as urgent nuclear data requirements by the Nuclear Energy Agency [22] have been performed. As an example, the measurement of the ${ }^{238} \mathrm{U}$ radiative capture cross-section [23] and of the ${ }^{240,242} \mathrm{Pu}(\mathrm{n}, f)$ cross-section [24, 25], with the purpose of obtaining a very high accuracy to solve present inconsis- 
tencies and improve the performances of new reactors. All these measurements have been performed in EAR1, but the ${ }^{240} \mathrm{Pu}(\mathrm{n}, f)$ one has suffered from several problems due to the damage of the MicroMegas detector caused by the very high $\alpha$-activity of the sample. For this reason the measurement has been repeated in 2014 exploiting the n_TOF EAR2, which has allowed to reduce the measurement time, thus preventing the damage of the detector.

The experimental campaign devoted to Nuclear Astrophysics is focused mostly on neutron magic nuclei, which act as bottle neck for the flow of s-process. Branching point isotopes for the s-process nucleosynthesis chain, i.e. those isotopes for which the time scale of neutron capture reactions and $\beta$ decays are comparable, have also been investigated. Among them, in 2012 the capture cross-section of ${ }^{63} \mathrm{Ni}$, which is the first branching point of the s-process nucleosynthesis chain, has been measured for the first time in a wide energy range from thermal neutron energies up to $200 \mathrm{keV}$ [26]. In total the capture kernels of 12 (new) resonances were determined, and stellar model calculations show that the new data have a significant effect on the s-process production of ${ }^{63} \mathrm{Cu},{ }^{64} \mathrm{Ni}$, and ${ }^{64} \mathrm{Zn}$ in massive stars.

In addition, isotopes of special interest such as the osmium involved in the so-called cosmic clock have been studied at $n \_$TOF. The clock is based on the extremely long half-life of ${ }^{187} \operatorname{Re}\left(\tau_{1 / 2}=43.3\right.$ Gyr), decaying to ${ }^{187} \mathrm{Os}$, and on the fact that ${ }^{186} \mathrm{Os}$ and ${ }^{187} \mathrm{Os}$ are shielded against direct r-process production. Thanks to the well established s-process abundances of the ${ }^{186} \mathrm{Os}$ and ${ }^{187} \mathrm{Os}$, the age of the Universe can be inferred, in the the Re/Os clock, by the enhancement in the abundance of ${ }^{187}$ Os due to ${ }^{187} \mathrm{Re} \rightarrow{ }^{187}$ Os decay. The neutron capture cross sections of ${ }^{186,187,188}$ Os have been measured at the n_TOF facility with improved accuracy and over a wide energy range of neutron energies from $1 \mathrm{eV}$ to $1 \mathrm{MeV}$. Using the $\mathrm{n}_{-} \mathrm{TOF}$ results within a schematic model that assumes an exponentially decreasing production rate for ${ }^{187} \mathrm{Re}$, an age of the Universe of $15 \pm 2$ Gyr was obtained from the $\mathrm{Re} / \mathrm{Os}$ cosmo-chronometer. More details can be found in Ref. [27-29].

With the construction of EAR2 the possibility to measure highly radioactive isotopes has been opened. In particular, for the first time the ${ }^{7} \mathrm{Be}(\mathrm{n}, \alpha)$ cross section has been measured in a wide energy range. This enters in the so-called "cosmological ${ }^{7} \mathrm{Li}$ problem" in Big Bang Nucleosynthesis (BBN), with the aim of lowering the uncertainty related to nuclear physics inputs in the BBN calculations. The challenges of the measurement, related to the low $(\mathrm{n}, \alpha)$ cross section and the very high specific activity of ${ }^{7} \mathrm{Be}$, have required a comprehensive study on its feasibility together with the development of a new Si-based detector.

The characteristics of the n_TOF facilities could be exploited also for measurements providing valuable information on basic nuclear physics quantities, such as levels densities, $\gamma$-ray strength functions and angular distributions. As an example, the study of $(\mathrm{n}, f)$ reactions with PPAC detectors provides valuable information on the angular distribution of the fission fragments [30]. Moreover, the high energy resolution of the facility allows a statistical analysis of the resonances obtained in capture measurements, which can result in important constraints both for Astrophysics and Nuclear Physics models. In the next paragraph the ${ }^{25} \mathrm{Mg}(\mathrm{n}, \gamma)$ measurement is illustrated as a significant example of the study of the level structure of the compound nucleus.

\subsection{The study of ${ }^{25} \mathrm{Mg}+\mathrm{n}$ reaction}

Elements heavier than iron are produced in stellar environments through the balance of neutron capture and $\beta$-decay processes. About half of these elements appear through the nucleosynthesis chain of the so called s-process (i.e. slow neutron capture process) [31]. Although most of the astrophysical sites of stellar nucleosynthesis and the nuclear processes involved have been identified, important physical and nuclear details are still largely unexplained, thus hindering a comprehensive understanding of the origin of the elements. In this view, an accurate knowledge of the nuclear reaction rates 

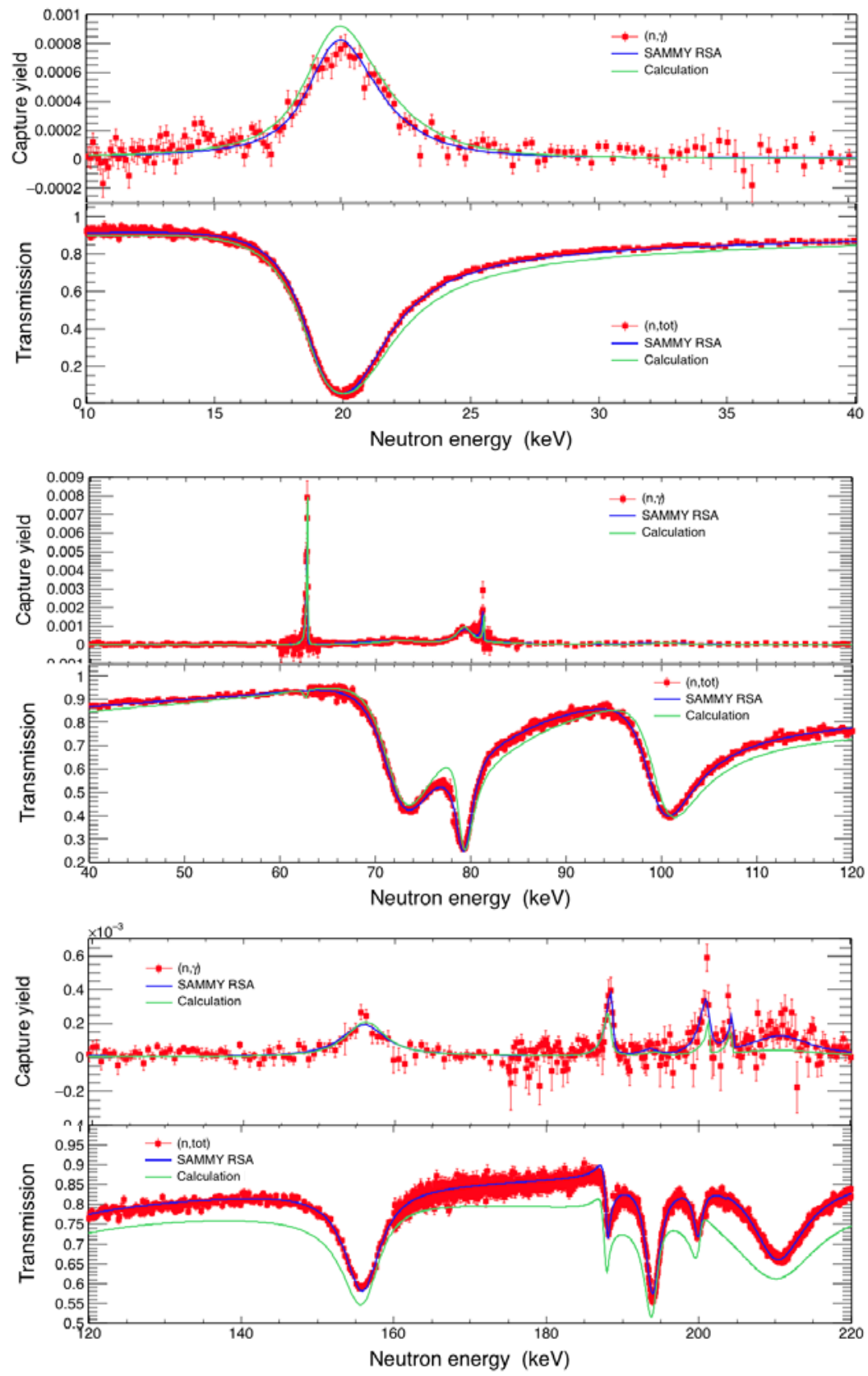

Figure 4. Comparisons between capture and transmission data in different energy ranges. The SAMMY RSA best fit curve is shown (blue line) together with the calculation performed using the parameters from the evaluation by Koehler [36] (green line). 
at stellar energy (i.e. $\mathrm{keV}$ energy region) is fundamental to constrain theoretical predictions of stellar models.

The $(\alpha, n)$ reaction on ${ }^{22} \mathrm{Ne}$ is the major astrophysical neutron source of the s process in massive stars and in intermediate AGB stars, while it is partially activated in low mass AGBs. Despite lots of attempts (see Ref. [32] and reference therein), direct measurements in the energy range of astrophysical sites are extremely difficult, mostly because of the extremely low cross section of the reaction at these energies and of cosmic-ray induced background. Data present in literature are therefore not accurate or even lacking, with the consequence that the ${ }^{22} \mathrm{Ne}(\alpha, \mathrm{n})^{25} \mathrm{Mg}$ reaction rate is very uncertain. This reaction rate at $\mathrm{s}$ process temperature depends on the level structure of the compound nucleus ${ }^{26} \mathrm{Mg}$ above the $\alpha$ threshold $(Q=10.615 \mathrm{MeV})$, close to the neutron threshold $\left(S_{n}=11.093 \mathrm{MeV}\right)$. Information on the properties of these poorly known states, especially on their spin-parity $J^{\pi}$, can be obtained from the ${ }^{25} \mathrm{Mg}(\mathrm{n}, \gamma)$ reaction by selecting only those states which can be populated by ${ }^{22} \mathrm{Ne}+\alpha$ reaction. For ${ }^{22} \mathrm{Ne}$ and $\alpha$ particles $J^{\pi}=0^{+}$, and therefore only natural parity states $\left(0^{+}, 1^{-}, 2^{+}, \ldots\right)$ can participate in the ${ }^{22} \mathrm{Ne}(\alpha, \mathrm{n})^{25} \mathrm{Mg}$ reaction, which correspond to a subset of ${ }^{26} \mathrm{Mg}$ states populated in the ${ }^{25} \mathrm{Mg}(\mathrm{n}, \gamma)$ reaction. In this context, it is crucial to have a precise assigment of the spin-parity numbers to each state studied in ${ }^{25} \mathrm{Mg}+\mathrm{n}$ reaction.

To this aim, the measurement of the ${ }^{25} \operatorname{Mg}(n, \gamma)$ cross section has been performed at $n \_$TOF in 2012, and in addition the total cross section on ${ }^{25} \mathrm{Mg}$ has been measured at the EC-JRC-IRMM facility GELINA in Belgium [33].

For the capture measurement, a low solid angle detection system composed of two $\mathrm{C}_{6} \mathrm{D}_{6}$ scintillators has been used. As previously pointed out, these detectors have a very low sensitivity to $\gamma$-rays induced by scattered neutrons, being therefore optimized to investigate light isotopes such as the ${ }^{25} \mathrm{Mg}$. The total energy detection technique has been used, in combination with the pulse-height weighting method [34] to obtain the required proportionality between the the efficiency of the detection system and the total radiative energy emitted by the capture event. The measurement has been carried out in EAR1, where single resonances could be resolved up to about $500 \mathrm{keV}$.

The experimental yield has been analyzed with the resonance shape analysis (RSA) code SAMMY [35] to obtain the parametrization of the capture cross-section in terms of resonance parameters. A simultaneous resonance shape analysis of both capture and transmission data is performed, which leads to much more precise resonance parameters as if the two data sets would have been analyzed separately. In Fig. 4 comparisons between capture and transmission data in different energy ranges are shown. The calculation performed using the parameters from the evaluation by Koehler [36] is shown as well on top of the data, together with the SAMMY best fit. It is evident from the figures that the new capture and transmission measurements call for a major revision of the evaluations, which suffered from several problems mainly due to impurities in the samples analyzed (see Ref. [37] and references therein).

Thanks to the new high-quality data provided at n_TOF and GELINA, a preliminary analysis of the statistical properties of the resonances has pointed out that for the level at $E_{R}=79 \mathrm{keV}$ the spin parity should change from $J^{\pi}=3^{+}$to $J^{\pi}=3^{-}$. If this would be confirmed, this state will become a natural-parity one and must be added to the number of states that can be populated by the ${ }^{22} \mathrm{Ne}(\alpha, \mathrm{n})$ reaction, affecting therefore its rate.

\section{Conclusion}

The $n$ _TOF facility became operative at CERN in 2001, and since then it is at the cutting edge for neutron induced reaction measurements. Since July 2014 a second experimental area (EAR2) has been built $20 \mathrm{~m}$ above the spallation target, with unique characteristics which made it a powerful tool 
for measurements of highly radioactive and of low-mass samples, and of very low cross sections. This new measuring station is complementary to the existing first experimental area (EAR1), and the characteristics of both are extremely competitive in terms of flux intensity, energy range covered and energy resolution. Depending on the requirements of the reaction under study precise and accurate results can be achieved, and several detection systems are available to investigate (n, $\gamma)$, (n,cp) and (n, $f$ ) reactions. These cross sections play a fundamental role in nuclear technologies, nuclear astrophysics and fundamental nuclear physics, and significant results have been attained, relating to new reactor designs, to stellar and primordial nucleosynthesis models and to the investigation of the compound nucleus. Associated to the last field, a recent measurement on the ${ }^{25} \mathrm{Mg}$ capture cross-section has allowed an accurate study of the level structure of the ${ }^{26} \mathrm{Mg}$ compound nucleus, providing important constraints on levels which can be populated by the ${ }^{22} \mathrm{Ne}(\alpha, \mathrm{n})$ reaction, one of the two main neutron source of the s process.

\section{References}

[1] C. Rubbia et al., CERN/LHC/98-02, CERN (1998).

[2] The n_TOF Collaboration, CERN INTC-2002-037 (2003).

[3] http://home.web.cern.ch/about/updates/2013/02/long-shutdown-1-exciting-times-ahead.

[4] The n TOF Collaboration, CERN-INTC-2012-029 / INTC-O-015 (2012).

[5] The CERN Accelerator Complex, http://public.web.cern.ch/public/en/research/AccelComplexen.html.

[6] C. Guerrero et al., Eur. Phys. J. A 49 (2013) 27.

[7] C. Weiß et al., Nucl. Instr. and Meth. A 799 (2015) 90.

[8] M. Barbagallo et al., Eur. Phys. J. A 49 (2013) 156.

[9] A. Ferrari and P.R. Sala, Intermediate and High Energy Physics Models in FLUKA: Improvements, Benchmarks and Applications, Proc. Int. Conf. on Nuclear Data for Science and Technology, NDST-97, ICTP, Miramare, Trieste, Italy, (19-24 May 1997).

[10] J. Briesmeister, MCNP-A General Monte Carlo N-Particlke Transport Code-Version 4c2, LA13709-M (2000).

[11] J. Lerendegui-Marco et al., submitted to Eur. Phys. J. A.

[12] P.F. Mastinu et al., n_TOF-PUB-2013-002, CERN-n_TOF-PUB-2013-002 (2013).

[13] C. Guerrero et al., Nucl. Instr. and Meth. A 608 (2009) 424-433.

[14] Y. Giomataris et al., Nucl. Instr. and Meth. A 376 (1996) 29.

[15] C. Guerrero et el., Eur. Phys. J. A 48 (2012) 29.

[16] S. Andriamonje et al., Development and performance of Microbulk Micromegas detectors, Proceedings of the 1st International Conference on Micro-Pattern Gaseous Detectors, Crete, Greece, 2009.

[17] C. Paradela et al., Physical Review C 82 (2010) 034601.

[18] L. Tassan-Got et al., CERN-INTC-2006-016, INTC-P-209.

[19] M. Barbagallo, A. Musumarra et al., CERN-INTC-2012-029 / INTC-O-015 (2012).

[20] Generation IV International Forum (GIF), https://www.gen-4.org/gif/jcms/c_9260/Public.

[21] OECD Nuclear Energy Agency, Accelerator-Driven Systems (ADS) and Fast Reactor (FR) in advanced nuclear fuel cycles. A comparative study, 2002.

[22] NEA-HPRL, High Priority Request List, hwww.nea.fr/html/dbdata/hprli.

[23] F. Mingrone et al., Nuclear Data Sheets 119 (2014) 18.

[24] M. Calviani, E. Berthoumieux et al., CERN-INTC-2010-042 / INTC-P-280 (2010). 
[25] A. Tsinganis et al., Nuclear Data Sheets 119 (2014) 58.

[26] C. Lederer et al., Phys. Rev. Lett. 110 (2013) 022501.

[27] M. Mosconi et al., Phys. Rev. C 82 (2010) 015802.

[28] M. Mosconi et al., Phys. Rev. C 82 (2010) 015803.

[29] K. Fujii et al., Phys. Rev. C 82 (2010) 015804.

[30] D. Tarrio et al., Nucl. Instr. and Meth. A 743 (2014) 79-85.

[31] E.M. Burbidge, G.R. Burbidge, W.A. Fowler and F. Hoyle, Rev. Mod. Phys. 29 (1957) 547.

[32] M. Jaeger et al., Phys. Rev. Lett. 87 (2001) 202501.

[33] W. Mondelaers and P. Schillebeeckx, Notiziario 11 (2006) 19.

[34] U. Abbondanno et al., Nuclear Instruments and Methods in Physics Research A 521 (2004) 454.

[35] N. M. Larson, ORNL/TM-9179/R8, Oak Ridge National Laboratory, Oak Ridge, TN, USA (2008). Also ENDF-364/R2.

[36] P. E. Koehler, Phys. Rev. C 66, 055805 (2002).

[37] C. Massimi et al., Phys. Rev. C 85 (2012) 044615. 